\title{
STRUCTURE-FROM-MOTION FOR CALIBRATION OF A VEHICLE CAMERA SYSTEM WITH NON-OVERLAPPING FIELDS-OF-VIEW IN AN URBAN ENVIRONMENT
}

\author{
A. Hanel ${ }^{\mathrm{a}}$, U. Stilla ${ }^{\mathrm{a}}$ \\ ${ }^{a}$ Photogrammetry \& Remote Sensing, Technische Universitaet Muenchen, Germany - (alexander.hanel, stilla)@tum.de
}

Commission I, WG 9

KEY WORDS: vehicle cameras, camera calibration, structure from motion, bundle adjustment

\begin{abstract}
:
Vehicle environment cameras observing traffic participants in the area around a car and interior cameras observing the car driver are important data sources for driver intention recognition algorithms. To combine information from both camera groups, a camera system calibration can be performed. Typically, there is no overlapping field-of-view between environment and interior cameras. Often no marked reference points are available in environments, which are a large enough to cover a car for the system calibration. In this contribution, a calibration method for a vehicle camera system with non-overlapping camera groups in an urban environment is described. A-priori images of an urban calibration environment taken with an external camera are processed with the structure-frommotion method to obtain an environment point cloud. Images of the vehicle interior, taken also with an external camera, are processed to obtain an interior point cloud. Both point clouds are tied to each other with images of both image sets showing the same real-world objects. The point clouds are transformed into a self-defined vehicle coordinate system describing the vehicle movement. On demand, videos can be recorded with the vehicle cameras in a calibration drive. Poses of vehicle environment cameras and interior cameras are estimated separately using ground control points from the respective point cloud. All poses of a vehicle camera estimated for different video frames are optimized in a bundle adjustment. In an experiment, a point cloud is created from images of an underground car park, as well as a point cloud of the interior of a Volkswagen test car is created. Videos of two environment and one interior cameras are recorded. Results show, that the vehicle camera poses are estimated successfully especially when the car is not moving. Position standard deviations in the centimeter range can be achieved for all vehicle cameras. Relative distances between the vehicle cameras deviate between one and ten centimeters from tachymeter reference measurements.
\end{abstract}

\section{DRIVER OBSERVATION FOR DRIVER INTENTION RECOGNITION}

One of the big goals in the automotive industry is to reduce the number of traffic fatalities to zero (Volvo Vision 2020 (Samuelsson, 2017)). An important part on this way is to know the intention of a car driver for the next seconds in advance. Currently available cars are therefore equipped with environment cameras to collect information about other traffic participants around the own car. For example, environment cameras can capture a preceding car slowing down on the rightmost lane and having activated the right turn indicator. Advanced driver assistance systems can use driver intention recognition algorithms to anticipate, that the preceding car driver wants to turn right.

To anticipate the intention of the own car driver, interior cameras observing his behavior (figure 1) can be used in addition. Their images can be used to extract features about the driver's head movement and his gaze direction. The intention recognition can be made more reliable, if information from the environment and interior cameras is combined. For example, combined evaluation allows to evaluate, whether the driver has noticed the slower car in front of him or is distracted by the car radio, for example. Therefore, his head orientation and gaze direction have to be linked to the position of the other car relative to his car. Basis for this geometric link is the known relative position and orientation (pose) of the interior and exterior vehicle cameras to each other. A system calibration for the vehicle cameras can be used to estimate these parameters.

For calibration of a vehicle camera system, a calibration environment large enough to contain a car is required, as well as the

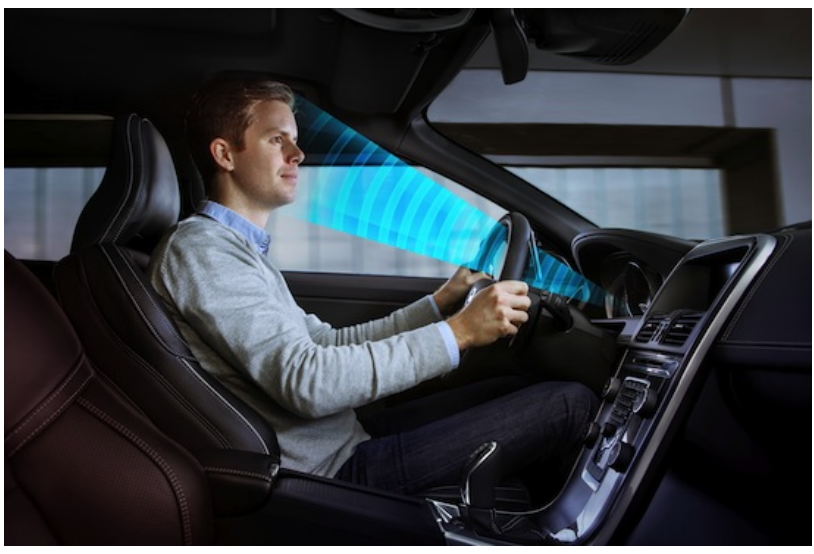

Figure 1: A driver camera can be used to extract features from images to recognize the driver intention of the next seconds as an important milestone to increase traffic safety (Volvo, 2014).

car has to be able to drive into this environment. Urban structures, like parking garages or court yards, can be used for this purpose. On the one hand, in urban structures additionally placed photogrammetric reference marks cannot be used, as these structures are public or there is no permission from the owner. On the other hand, vehicle interior cameras can see almost nothing than the interior space of a car. Large parts of the interior of a car, like the window pane or the seats cannot be used to place reference marks. Therefore, a calibration approach using unmarked reference points is required. For example, feature points extracted from images and their $3 \mathrm{~d}$ coordinates calculated automatically can be used as reference. 
$3 \mathrm{~d}$ coordinates of reference points can be calculated automatically by triangulating image features of urban structures shown in multiple images. However, as the vehicle interior cameras are showing nearly only the interior space of the car, reference information of the urban structures is not available for them. Other reference information has to be used for these cameras, and the reference information for both environment and interior cameras has to be linked together for a system calibration.

As costs are a very important factor in the automotive industry, the number of cameras is kept small. This leads in addition to non-overlapping fields-of-view, making the camera system calibration using tie points in overlapping image parts impossible. Due to their wide field-of-view and their low costs, so called "action cameras" can cover a huge part of the environment around a car despite their small number. Therefore, it has to be considered, that the wide field-of-view might cause large image distortions requiring reliable single camera calibration.

In addition, the camera mounting on the window panes of the car might not be rigid over time caused by mechanical movements during a car drive. To check the vehicle camera poses again and again, the system calibration has to be repeated from time to time, requiring a calibration process which can be performed with low effort, for example before and after every video recording drive.

The camera system calibration provides information about the relative poses of the vehicle cameras. To know, whether a pedestrian shown in one of the environment camera images is behind or in front of the own car, the camera poses have to be linked to the pose of the car. This can be done by transforming the camera poses into a vehicle coordinate system, which describes the movement direction of the car. As ground control points for the transformation, physical points on the car surface corresponding to the vehicle coordinate system have to be chosen.

\section{STATE OF THE ART}

\subsection{Camera calibration}

Geometric calibration is performed on single cameras to estimate the interior orientation as well as image distortion parameters (Brown, 1971), (Fraser, 2013), (Hartley and Zisserman, 2004). Different models, like perspective pinhole models or fisheye models can be used (Abraham and Förstner, 2005), (Schwalbe, 2005). Geometric calibration can be also performed on multiple cameras simultaneously to estimate the relative poses of the cameras (Gruen and Beyer, 2001), wherefore typically calibration patterns are used. For calibration of commercial vehicle camera systems, manufacturer-specific calibration patterns are required, which are only available in calibration laboratories (glassBYTEs.com, 2015). Recently published approaches do not require calibration patterns anymore for system calibration of rigid camera systems (Esquivel et al., 2007), (Kazik et al., 2012), (Schneider and Förstner, 2013).

\subsection{Structure from motion}

The structure-from-motion ( $S f M)$ method is used for $3 \mathrm{~d}$ reconstruction ('structure') of objects shown in an image set, whereby the movement of the camera between different images is used as stereo base line (Koenderink et al., 1991), (Sturm and Triggs, 1996). The usual pipeline (e.g. (Häming, 2010)) is to extract features from images and to match them with each other. Camera poses are estimated and $3 \mathrm{~d}$ points triangulated and optimized in a bundle adjustment. There are different software toolboxes available for structure-from-motion, a well known example from the literature is VisualSFM (Wu, 2007), (Wu, 2011), (Wu et al., 2011).

Camera system calibration and structure from motion can be combined by using reconstructed $3 \mathrm{~d}$ points of a space to calibrate a camera system (Hanel and Stilla, 2017). The method in the cited paper requires, that each camera can see the $3 \mathrm{~d}$ reference points, which might not be true for vehicle interior cameras.

\subsection{Street scene datasets}

Like for the vehicle camera system proposed in this contribution, vehicle cameras are often used to collect street scene data. Among others, typically vehicle environment cameras (e.g. for datasets Kitti (Geiger et al., 2012), Cityscapes (Cordts et al., 2016)) are used for data collection. For the mentioned datasets, the interior and exterior calibration parameters of the camera systems are provided. Vehicle interior cameras are used only for some datasets, e.g. provided by Brain4Cars (Jain et al., 2015), while this dataset does not provide calibration parameters for the cameras.

\section{VEHICLE CAMERA SYSTEM CALIBRATION IN AN URBAN ENVIRONMENT}

In this section, a method (figure 2) to calibrate a vehicle camera system with environment and interior cameras without overlapping fields-of-view using unmarked reference points from urban structures and from the vehicle interior is described.

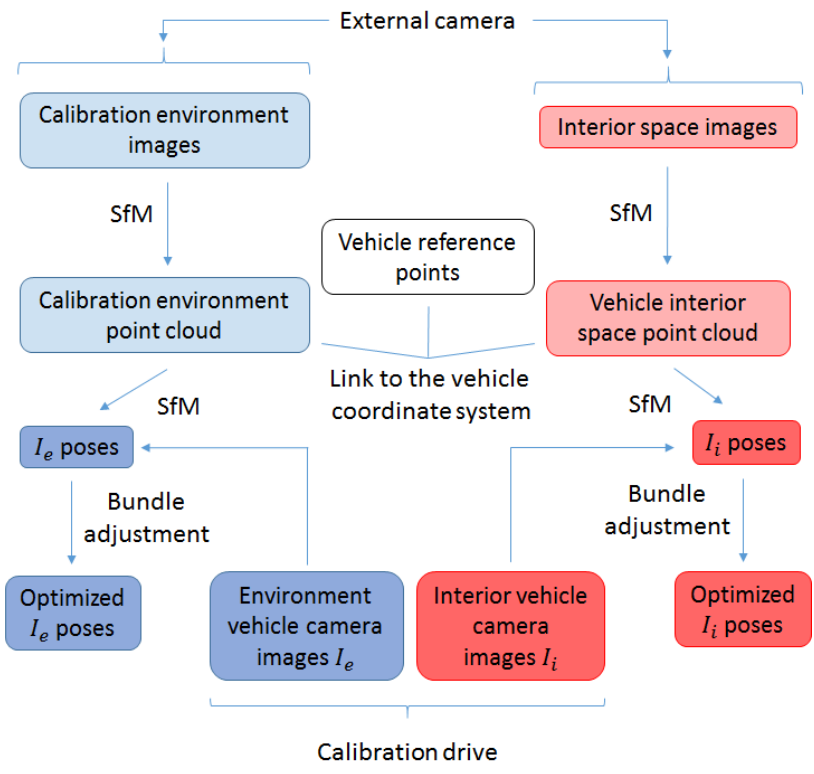

Figure 2: Process workflow to estimate the poses of vehicle environment $I_{e}$ and vehicle interior cameras $I_{i}$ with non-overlapping fields-of-view in an urban environment.

For pre-processing, each camera is calibrated separately in a laboratory environment to undistort all images. The main process chain can be divided into an a-priori part, which has to be done once with images of an external photographic camera before calibrating the vehicle camera system and the calibration part, which is repeated every time the vehicle camera system should be calibrated.

In the a-priori part, a point cloud of the calibration environment (environment point cloud) is created using structure-from-motion 
with images of urban structures in the calibration environment (environment images). Another point cloud of the interior space of the vehicle (interior point cloud) is created using structurefrom-motion with images of the vehicle interior (interior images). Both point cloud coordinate systems are connected with common reference points and transformed into a vehicle coordinate system using reference points describing the vehicle movement.

In the calibration part, the poses of the vehicle cameras are estimated. Images of the vehicle environment cameras are matched with the environment images to use the $3 \mathrm{~d}$ points of the environment point cloud as ground control points for pose estimation of the vehicle environment cameras in the vehicle coordinate system. The same way, images of the vehicle interior cameras are matched with interior images and the $3 \mathrm{~d}$ points of the interior point cloud used for pose estimation of the vehicle interior cameras in the vehicle coordinate system. Finally, all vehicle camera poses are adjusted using separate bundle adjustments for environment and for interior cameras.

\subsection{Single camera calibration}

The single camera calibration is performed for each camera to reduce the degrees of freedom for the point cloud creation and the pose estimation. The following process is used: A planar calibration pattern with photogrammetric marks is captured in images with different camera positions, orientations and distances to the pattern plane. The interior orientation parameters (focal length in $x / y$ direction, principal point in $x / y$ direction) as well as radial and tangential image distortion parameters are estimated (Conrady, 1919), (Brown, 1971).

The following radial distortion model is used (equations 1,2 ):

$$
\begin{aligned}
& x_{\text {dist }, \text { rad }}=x \cdot\left(1+k_{1} r^{2}+k_{2} r^{4}+k_{3} r^{6}\right) \\
& y_{\text {dist }, \text { rad }}=y \cdot\left(1+k_{1} r^{2}+k_{2} r^{4}+k_{3} r^{6}\right)
\end{aligned}
$$

with $x_{d i s t, r a d}, y_{d i s t, r a d}$ as distorted image coordinates, $x, y$ as undistorted image coordinates, $r$ as radial distance of $x, y$ from the principal point and $k_{1}, k_{2}, k_{3}$ as radial distortion parameters.

The following tangential distortion model is added (equations 3 , 4):

$$
\begin{aligned}
x_{d i s t} & =x_{d i s t, r a d}+\left[2 p_{1} x y+p_{2}\left(r^{2}+2 x^{2}\right)\right] \\
y_{d i s t} & =y_{d i s t, r a d}+\left[p_{1}\left(r^{2}+2 y^{2}\right)+2 p_{2} x y\right]
\end{aligned}
$$

with $p_{1}, p_{2}$ as tangential distortion parameters.

To calculate the desired camera parameters, the sum over all images and pattern points of the reprojection errors from $3 d$ pattern points into the images is minimized using the LevenbergMarquardt optimization. The images of each camera are undistorted using the parameters obtained by camera calibration before processing the following steps.

\subsection{Point cloud creation}

This and the following parts of the method are the a-priori part of the processing, i.e. they have to be done once to be able to calibrate the vehicle camera system. The images used in this part are taken with an additional camera, not by the vehicle cameras.

In the a-priori part of the processing, the environment point cloud of urban structures (e.g. building walls, signs) in the calibration environment has to be created, as well as the interior point cloud of the interior of the camera-carrying vehicle has to be created. For the environment point cloud, a high number of overlapping environment images is taken within the calibration environment SIFT features (Lowe, 1999) are extracted from these images and used to match the images with each other. The point cloud is created using the structure-from-motion method, providing $3 \mathrm{~d}$ point coordinates for the SIFT feature matches and also 3d coordinates for the camera poses. Only $3 \mathrm{~d}$ points with matches between at least three images are accepted for further processing.

For the interior point cloud, interior images are taken in the interior space of the vehicle and also the SfM method applied to get the $3 \mathrm{~d}$ point coordinates for the SIFT feature matches.

Both image sets need to have overlapping images showing the same objects to transform one point cloud coordinate system into the other.

\subsection{Transformation into the vehicle coordinate system}

Both point cloud coordinate systems are transformed into the vehicle coordinate system to create a link between the estimated vehicle poses (see subsections $3.4,3.5$ ) to the driving direction of the camera-carrying car.

This step consists of two parts: Connecting the two point cloud coordinate systems to each other and transforming the connected point cloud coordinate system of the into the vehicle coordinate system.

For step one, $3 \mathrm{~d}$ coordinates of common reference points shown in both environment images and interior images are used. The interior point cloud is transformed into the environment point cloud coordinate system using a similarity transform (equation 5):

$$
x^{\prime}=s \cdot R \cdot x+t
$$

with $x^{\prime}, x$ target and source $3 \mathrm{~d}$ point coordinates, and the parameters $s$ as scale, $R$ as rotation matrix and $t$ as translation vector.

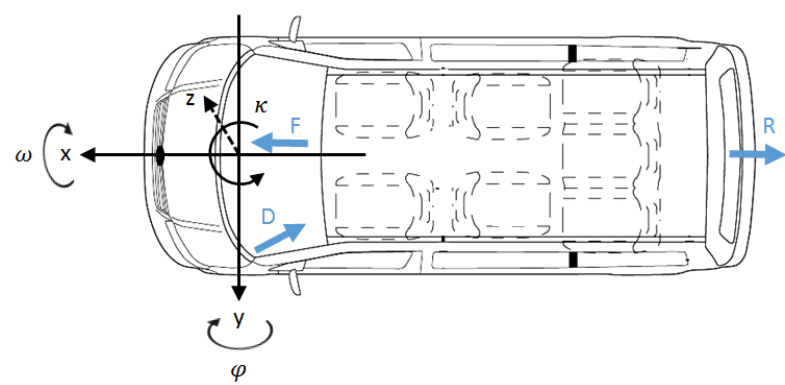

Figure 3: Vehicle coordinate system. Origin is the center of the front axle. $Y$-axis is equal to front axle, $\mathrm{x}$-axis through front and rear axle and orthogonal to $y$-axis. Z-axis is orthogonal to the other two axes. Vehicle cameras of the test car are shown in blue $(\mathrm{F}=$ front, $\mathrm{D}=$ driver, $\mathrm{R}=$ rear), position of the cameras at the arrow tail, viewing direction like arrows (Volkswagen, 2017).

For step two, the vehicle coordinate system (figure 3 ) is defined by both axles of the car. It is assumed, that both axles are at the same height above ground, i.e. the vehicle coordinate system is parallel to the ground. The center of the front axle is defined as the origin of the vehicle coordinate system. The $\mathrm{x}$-axis goes through the center of both the front and rear axle, pointing in driving direction, assuming that this definition describes the driving direction of the car. The y-axis is equal to the front axle, pointing to the left side of the car. The z-axis is orthogonal to the two other axes and pointing away from the ground.

A similarity transformation is used as transformation method, the transformation parameters are estimated with RANSAC and a least-squares estimation. 
The transformation uses the rim centers (figure 4) of the four wheels as ground control points. It is assumed, that the rim centers are the extension of the car axles. As modern car wheels have independent suspension, the camera-carrying chassis is only parallel to the rim centers, if the car is standing on a level plane.

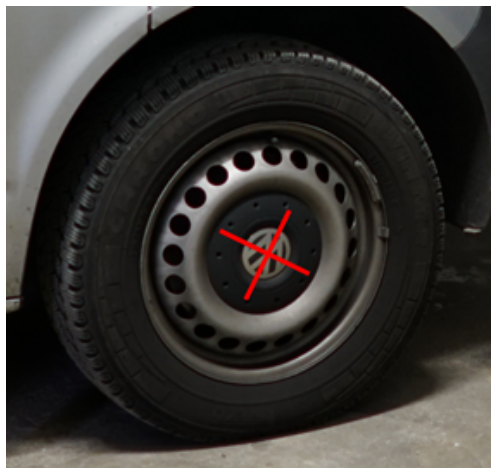

Figure 4: Rim centres (red cross) are used as ground control points for the transformation into the vehicle coordinate system. The emblem of the car manufacturer is often placed in the rim center providing a clear defined central point.

The image points showing the rim centers in environment images are used to calculate the $3 \mathrm{~d}$ coordinates of the rim centers in the point cloud coordinate system. The $3 \mathrm{~d}$ coordinates of the rim centers in the vehicle coordinate system can be calculated for example from a blueprint of the car manufacturer providing length information for relevant vehicle parts, like the wheel base.

The transformation is applied to the connected point cloud coordinate system, providing the point clouds in the vehicle camera system.

\subsection{Pose estimation of vehicle environment cameras}

This and the following parts of the method are the calibration part of the processing chain, i.e. they have to be performed every time the calibration of the vehicle cameras should be repeated.

Videos of the vehicle cameras recorded during a calibration drive of the car within the calibration environment are used for pose estimation of the vehicle cameras with the SfM method. SIFT features are extracted from the vehicle environment camera images, the features used to match the vehicle camera images to each other and to the environment images. The matches between the environment images and the vehicle camera images allow to use the $3 \mathrm{~d}$ points of the environment point cloud as ground control points to estimate the poses of the vehicle environment cameras.

\subsection{Pose estimation of vehicle interior cameras}

For pose estimation of the vehicle interior cameras, videos recorded at the same time as the videos of the environment cameras (subsection 3.4) are used. Also SIFT features are extracted to match the vehicle interior camera images to each other and to the interior images. Thereby, the interior point cloud is used as reference information to estimate the poses of the vehicle interior cameras.

\subsection{Pose optimization by bundle adjustment}

The poses of the vehicle environment cameras and the vehicle interior cameras are optimized in separate bundle adjustments. As there is no overlap between the two image sets of the vehicle cameras, two separate bundle adjustments are required.
The environment images and the images of the vehicle environment cameras are processed together in one bundle adjustment. The $3 \mathrm{~d}$ coordinates of the point cloud and the corresponding image coordinates are introduced as adjustable parameters. A-priori values for these parameters are obtained from the environment point cloud (subsection 3.2) and the pose estimation (subsection 3.4). The exterior orientations of the cameras are introduced as adjustable parameters, too; the a-priori values for the exterior orientations are taken from the poses estimated with SfM (subsections 3.2,3.4). The interior orientations of the cameras and their distortion parameters are kept fixed on the values from single camera calibration (subsection 3.1), as the single camera calibration can provide higher certainty for these values than the SfM method.

Bundle adjustment is done by a least-squares adjustment based on collinearity equations (equations 6,7 ):

$$
\begin{array}{r}
x_{i j}=x_{0 j}-c_{j} \\
\frac{R_{11 j}\left(X_{i}-X_{0 j}\right)+R_{21 j}\left(Y_{i}-Y_{0 j}\right)+R_{31 j}\left(Z_{i}-Z_{0 j}\right)}{} \\
\hline R_{13 j}\left(X_{i}-X_{0 j}\right)+R_{23 j}\left(Y_{i}-Y_{0 j}\right)+R_{33 j}\left(Z_{i}-Z_{0 j}\right) \\
y_{i j}=y_{0 j}-c_{j} \\
\frac{R_{12 j}\left(X_{i}-X_{0 j}\right)+R_{22 j}\left(Y_{i}-Y_{0 j}\right)+R_{33 j}\left(Z_{i}-Z_{0 j}\right)}{R_{13 j}\left(X_{i}-X_{0 j}\right)+R_{23 j}\left(Y_{i}-Y_{0 j}\right)+R_{33 j}\left(Z_{i}-Z_{0 j}\right)}
\end{array}
$$

with $X, Y, Z$ as $3 \mathrm{~d}$ world coordinates, $x, y$ as image coordinates, $x_{0}, y_{0}, c$ as parameters of the interior orientation, $X_{0}, Y_{0}, Z_{0}$ as the camera position in world coordinates and $R$ describing the camera orientation. Index $i$ represents ground control points, index $j$ represents images.

For the least-squares adjustment, the residuals between the measured image coordinates and the image coordinates calculated using the estimated camera parameters and point coordinates are minimized.

The interior images and the images of the vehicle interior cameras are processed together in another bundle adjustment. Constraints for vehicle camera poses are not introduced into the bundle adjustment, as the camera system might not be rigid due to the non-rigid mounting with suction pads on window panes and mechanical movements during the calibration drive.

\section{TEST DATA ACQUISITION AND PROCESSING}

The calibration environment is within an underground car park. The environment itself has a parking lot on one side of a driveway, and two parking lots on the other side. The car park is built of concrete, in some parts painted white, and therefore without strong textures. Some signs are painted onto the walls. The only illumination source is a neon lamp at the ceiling, providing darker light than natural daylight. The car park is chosen, as the illumination does not change over time and there are no moving objects (e.g. tree leafs, persons). The experiments are conducted in the late evening when the car park is empty and there are no cars leaving or entering the garage.

A Volkswagen Transporter 5 van is used as test car. Two environment cameras and one interior driver camera are installed in the car. The positions of the cameras are chosen to cause the least possible occlusion for the field of view for the car driver. The first environment camera is mounted on the windshield and looking at the area in front of the car (figure 5). The second environment camera is mounted on the rear window and looking at the area behind the car (figure 6). The interior camera is also mounted on the windshield and looking at the driver's seat (figure 5). 


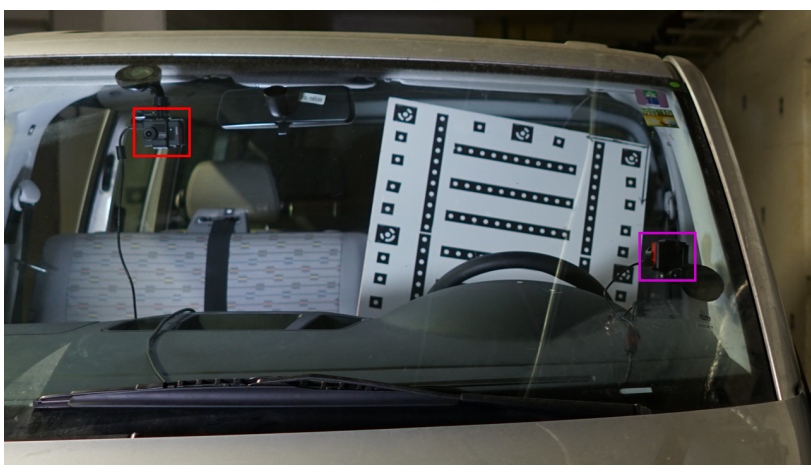

Figure 5: View onto the test car windshield against the driving direction. The front environment camera (red rectangle) is looking at the area in front of the car and the interior driver camera (purple rectangle) is looking at the car driver. Photogrammetric marks on the calibration pattern are used as ground control points to link the driver camera to the vehicle coordinate system.

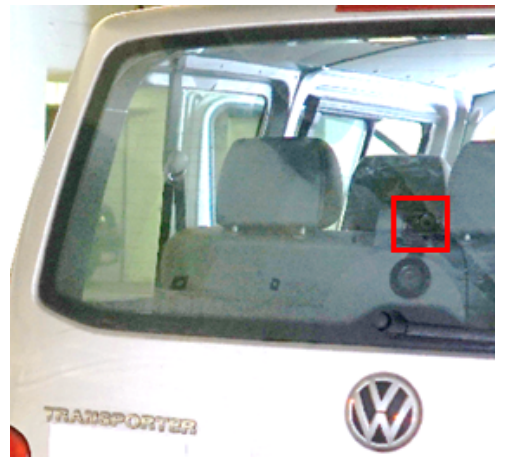

Figure 6: View onto the rear window pane of the test car. The rear environment camera (red rectangle) is looking at the area behind the car. Image brightness increased strongly for better visualization.

A-priori to the vehicle camera calibration, 143 images of the calibration environment are taken with an external DSLR camera (table 1) to create the environment point cloud with the SfM toolbox VisualSFM. In some of these images, photogrammetric marks placed on walls of the calibration environment are shown. 162 images are taken of the front seat row in the test car to create the interior point cloud. Some of these images contain a planar pattern with photogrammetric marks.

\begin{tabular}{l|l} 
Camera & Nikon D3 \\
Lens & $24 \mathrm{~mm}$ fixed focal length \\
Geometrical resolution & $4,256 \times 2,832 \mathrm{px}$ \\
Settings & $\begin{array}{l}\text { ISO 200, focus infinite, f/8, } \\
\text { exposure time variable }\end{array}$
\end{tabular}

Table 1: Technical specifications and image acquisition parameters of the external DSLR camera used for the environment images (Nikon, 2007).

The photogrammetric marks placed on walls in the calibration environment and the marks on the calibration pattern in the car are used as reference points for the transformation of the interior point cloud into the environment point cloud coordinate system. The $3 \mathrm{~d}$ coordinates of all marks are calculated from tachymeter (model: Leica TS02) measurements and used for the transformation together with the corresponding image coordinates measured (software: AICON 3D Studio (Schneider et al., 2017)) in the environment images and interior images, respectively. In general, the tachymeter measurements are not necessary to connect the two point clouds (cf. subsection 3.3), but are used here for evaluation purposes. For the transformation into the vehicle co-

\begin{tabular}{l|l} 
Camera & Garmin VIRB Ultra 30 \\
Lens & $2.73 \mathrm{~mm}$ fixed focal length \\
Geometrical resolution & $2,688 \times 1,512 \mathrm{px}$ \\
Temporal resolution & $30 \mathrm{fps}$ \\
Settings & ISO 800 , focus infinite, f/2.6
\end{tabular}

Table 2: Technical specifications and video recording parameters of the action cameras used in the test car to record videos (Pemble, 2017).

ordinate system, $3 \mathrm{~d}$ coordinates of the rim centers are calculated from environment images. Scale can be introduced from vehicle blueprints with metric information of vehicle parts (e.g. wheel base, total length). Several points on each case of the vehicle cameras are measured with the tachymeter to evaluate the estimated poses.

To calibrate the vehicle cameras, 248 images are extracted from videos of each vehicle camera (table 2 ) of a calibration drive, one image per second. The test car is standing still or moving slowly $(<5 \mathrm{~km} / \mathrm{h})$ during the video recording. Temporal synchronization between the vehicle cameras is achieved with a short flashlight at the beginning of the video recording. Previous tests of the synchronization have shown a maximum time gap between the three cameras of 0.1 seconds. As the vehicle cameras should be used in future research to record street scenes, videos instead of images are used for the calibration. The images of the front and rear vehicle camera are matched with the environment images, the poses of the vehicle camera images estimated in the vehicle coordinate system and adjusted in the bundle adjustment. The images of the vehicle driver camera are matched with the interior images. To ensure, that features are only extracted from image parts showing the vehicle interior, the other image parts are excluded from the calculation. Another bundle adjustment is performed to optimize the estimated poses.

\section{RESULTS AND DISCUSSION}

In this section, the results of the single camera calibration, the point cloud creation and the vehicle camera pose estimation are shown and discussed.

Single camera calibration. The RMS values (table 3) calculated for the single camera calibration vary up to $2 \mathrm{px}$. The pinhole model of openCV (OpenCV, 2017) is used for calibration and image undistortion, as the Garmin cameras apply internally already an image distortion correction. So the pinhole model is applied to correct the remaining distortions, wherefore calibration with the openCV fisheye model has led to poor RMS values (factor 500 worse). The high RMS values (RMS $<1 \mathrm{px}$ is considered as 'low value') of the pinhole model might be caused by the wide-angle lens of the used Garmin action cameras (diagonal FOV $133.6^{\circ}$, (Pemble, 2017)); in addition, there is no information available about the quality of the internal image distortion correction. The difference of the RMS values for the different cameras might be explained by different poses of the pattern relative to each camera during calibration.

\begin{tabular}{l|l} 
Front camera & $1.97 \mathrm{px}$ \\
Driver camera & $0.91 \mathrm{px}$ \\
Rear camera & $0.36 \mathrm{px}$
\end{tabular}

Table 3: RMS values of the single camera calibration using a pinhole model for each vehicle camera.

Point clouds. The created environment point cloud consists of around 26,000 $3 \mathrm{~d}$ points and is representing mostly the walls in the calibration environment. Only few $3 \mathrm{~d}$ points are on the floor or on the ceiling. More than 600,000 SIFT features are extracted 
from the environment images, in average around 4,800 SIFT features per image. More than 126 thousand combined matches (i.e., independent whether a SIFT feature is found in two or more images, it is counted as one match) are calculated from all image pairs, cf. (Hanel and Stilla, 2017).

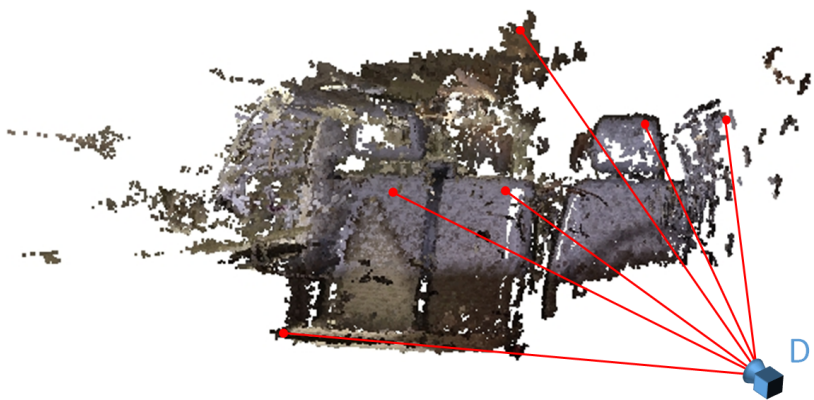

Figure 7: Point cloud of the front seat row of the test car, view from outside through the windshield to the interior. Three seats can be seen in the figure, the driver's seat is on the right side. $3 \mathrm{~d}$ points (examples: red circle) of this point cloud are used to estimate the pose of the interior driver camera $D$. In average, 180 points per image are used for pose estimation.

The interior point cloud (figure 7) consists of around 7,000 3d points and more than 43,000 combined matches. It shows the front seat row of the test car with the most $3 \mathrm{~d}$ points on the seat surface and a lower number of points on the ceiling of the car. The seats have a repetitive pattern surface, whose color differences are needed to extract image features and to find matches. The lower number of $3 \mathrm{~d}$ points of the interior point cloud compared to the environment point cloud might be drawn back to two aspects: The covered area is smaller and the illumination of the car interior is very poor, so that some areas appear dark in the images used for point cloud creation.

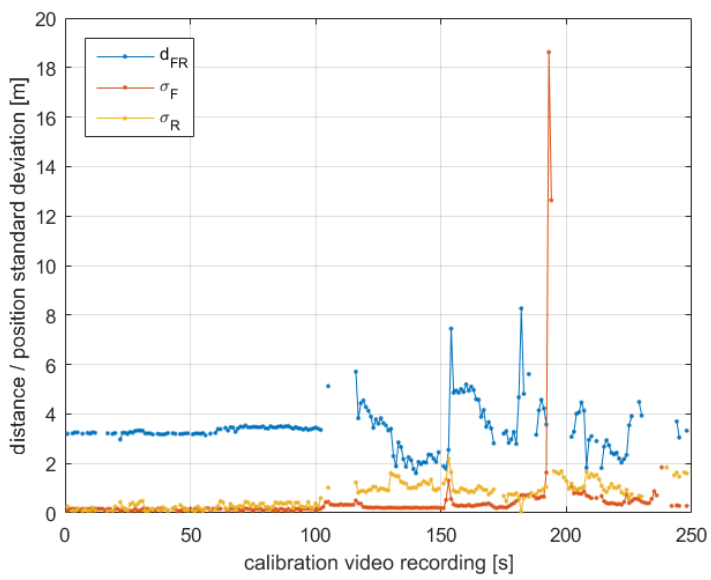

Figure 8: Distance $d_{F R}$ between vehicle front and rear camera plotted over the time of the calibration drive videos, as well as the position standard deviation of the vehicle front camera $\sigma_{F}$ and vehicle rear camera $\sigma_{R}$. Gaps in the plot occur, if a camera pose could not be estimated for a specific time point. The plot shows stable values while the vehicle is standing, a lower accuracy while the vehicle is moving. Line between discrete poses drawn for better visualization.

Vehicle poses. For the most consecutive images of the vehicle environment cameras, the poses can be estimated with reference information from the environment point cloud (figure 8). In the videos of the calibration drive, the vehicle is standing still until around second 100, afterwards it is moving within the calibration environment. Therefore, for the first 100 seconds, the pose estimation is stable for nearly all images and can be considered as successful, while afterwards the value variations increase and the estimation fails for some images (gaps in figure 8). Pose estimation fails especially, when the test car is moving into a part of the calibration environment with a low density of the environment point cloud, cf. (Hanel and Stilla, 2017). Large value variations might be explained by motion blur while driving, varying number and distribution of ground control points. Further, the link between the environment and interior vehicle cameras is only kept, while the vehicle is standing. Therefore, and for quality reasons, it should be considered to calibrate the cameras in future experiments only with images taken while the vehicle is standing.

For numeric evaluation, the distance $d_{F R}$ between the vehicle front and vehicle rear camera is calculated from the estimated poses (figure 8). For the first around 100 images, the distance varies within around $30 \mathrm{~cm}$, while the variations increase afterwards up to several meters. The position standard deviations $\sigma_{F}, \sigma_{R}$ (e.g. $\sigma_{F}=\left\|\sigma_{\mathbf{F}, \mathbf{V}}\right\|_{2}$ with $\sigma_{F, V}=\left[\begin{array}{lll}\sigma_{F, X} & \sigma_{F, Y} & \sigma_{F, Z}\end{array}\right]^{T}$ ) of the two vehicle environment camera positions are around 10 $\mathrm{cm}$ while the car is standing. The smaller variations of the standard deviation of the front camera compared to the rear camera might be explained by the higher number of $3 d$ points of the environment point cloud shown in the images of the front camera. At that time, the rear camera is closer to walls than the front camera, reducing the number of $3 \mathrm{~d}$ points shown in the images. For the time points the car is in movement, the values of the standard deviations as well as their variations increase. To conclude, the more stable distance values and standard deviations until second 100 show again the importance, that the test car is standing still at different positions during the calibration drive. Numeric evaluation based on the correlations between interior and exterior orientation parameters is not possible, as the interior parameters are kept fixed in the bundle adjustment due to their accurate estimation in the single camera calibration.

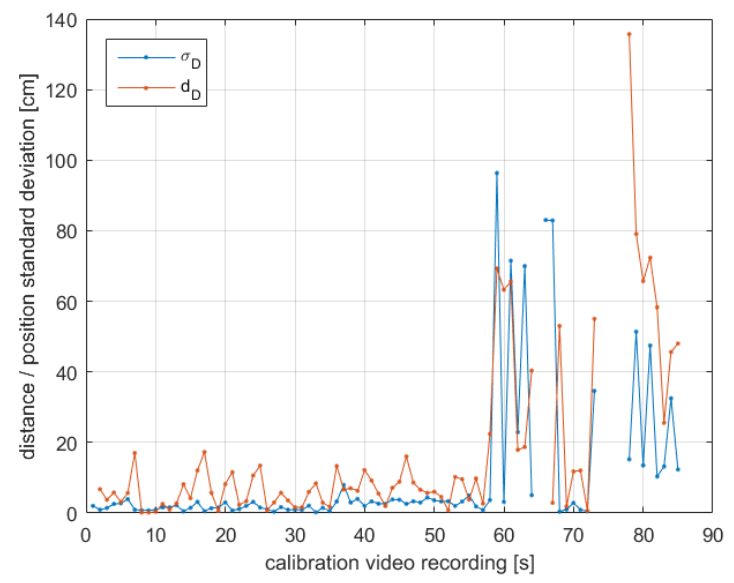

Figure 9: Variations $d_{D}$ of the estimated vehicle interior driver camera positions over the time of video recording, as well as the position standard deviation of the vehicle interior camera $\sigma_{D}$. Plotted over the time the vehicle is standing still during the calibration drive. Line between discrete poses drawn for better visualization.

The poses of the driver camera (figure 9) are calculated with ground control points from the interior point cloud. The position standard deviation varies between $10 \mathrm{~mm}$ and $20 \mathrm{~mm}$ for the first 60 seconds of the calibration video. In that time, the calibration pattern (figure 5) is placed in the car. No photogrammetric marks shown in the pattern are used for pose estimation, but it occludes a large part of the car interior from the point of view of 
the interior vehicle camera, leading to a reduced number of feature matches used for ground control points. Image noise caused by low light in the calibration environment might have also led to different SIFT features in different images, finding no matches to features detected in other images. The higher position standard deviation with values up to $1,000 \mathrm{~mm}$ after 60 seconds might be explained as a person enters the car at that time to drive it around within the calibration environment, causing further occlusions of the car seats in the driver camera images. The position differences show a similar trend than the position standard deviations, but with periodic value changes in the first 60 seconds. These observations should be investigated further to conclude on their reason.

A match matrix (showing the number of feature matches between all image pairs) between the a-priori interior images and the vehicle interior camera images shows, that there is only a small number of feature matches between the two mentioned image groups. This small number leads to a small number of ground control points for pose estimation, and therefore has negative influence on the position standard deviation. A possible explanation for the small match number is, that the strongly-textured car seats with a high number of possible features can only be seen in a small part of a vehicle interior camera image. A change in the position of this camera should be considered, requiring a trade-off with the occlusion of the field-of-view of the driver.

\begin{tabular}{l|r|r} 
& Median distance & Reference distance \\
Front - Rear & $321 \mathrm{~cm}$ & $323 \mathrm{~cm}$ \\
Front - Driver & $81 \mathrm{~cm}$ & $89 \mathrm{~cm}$ \\
Driver - Rear & $359 \mathrm{~cm}$ & $358 \mathrm{~cm}$
\end{tabular}

Table 4: Median and reference distances between the vehicle camera positions. Median is obtained from the calculated distances for all time points using the estimated camera positions. The reference distances are obtained from tachymeter measurements.

The median distance of the vehicle cameras positions to each other is compared to the distances from tachymeter measurements (table 4). The median is calculated for the distances between the camera positions while the test car is standing still during the calibration drive. As a tachymeter can only measure points on the camera case, but not the projection center, it is assumed, that the projection centers of the used Garmin action cameras are in the middle of the camera case in direction of the optical axis (no further manufacturer information available). The median distance between the front and rear camera deviates only two centimeters from the reference distance, while the deviation for the distance between front and driver camera is around $10 \mathrm{~cm}$. The smaller deviation between the vehicle environment cameras might be explained, because both camera poses are estimated using the same point cloud as reference information. In opposite, the front and driver camera poses are estimated with different point clouds, and an additional error might be caused by the transformation between the point clouds. The deviation for the driver and rear camera is only one centimeter, but it has to be mentioned, that the distance deviation is smaller than the calculated position standard deviations (figures 8,9 ).

\section{CONCLUSION}

In this contribution, a method for a vehicle camera system calibration using unmarked reference points has been proposed. The method can be used for vehicle environment and vehicle interior cameras with no overlapping fields-of-view. It is shown, that point clouds can be obtained for the environment around the vehicle as well as for its interior space. Both point clouds can be connected to each other using a similarity transform with reference points from objects shown in environment images and interior images as well. With a second similarity transformation, the point clouds are transformed into a vehicle coordinate system describing the movement direction of the car using reference points on the wheel rim centers. The poses of both groups of vehicle cameras can be estimated especially when the vehicle is standing still during a test drive using the $3 \mathrm{~d}$ points of the point clouds as reference information. The proposed method can be seen as suitable for an application in the field of driver intention recognition. For this application, the knowledge of the camera poses with a centimeter position accuracy is sufficient for combined information extraction about the traffic situation from vehicle environment and interior cameras.

For the future, a change of the position of the interior driver camera has to be considered. A position with a better view on welltextured surfaces like car seats might facilitate the feature extraction and therefore increase the accuracy of the estimated poses. Further research can also be done on validation of the estimated poses in long-time vehicle drives.

\section{REFERENCES}

Abraham, S. and Förstner, W., 2005. Fish-eye-stereo calibration and epipolar rectification. ISPRS Journal of Photogrammetry and Remote Sensing 59(5), pp. 278 - 288.

Brown, D. C., 1971. Close-range camera calibration. Photogrammetric Engineering 37(8), pp. 855-866.

Conrady, A. E., 1919. Decentred Lens-Systems. Monthly notices of the Royal Astronomical Society.

Cordts, M., Omran, M., Ramos, S., Rehfeld, T., Enzweiler, M., Benenson, R., Franke, U., Roth, S. and Schiele, B., 2016. The cityscapes dataset for semantic urban scene understanding. CoRR.

Esquivel, S., Woelk, F. and Koch, R., 2007. Calibration of a multi-camera rig from non-overlapping views. In: Proceedings of the 29th DAGM Conference on Pattern Recognition, SpringerVerlag, Berlin, Heidelberg, pp. 82-91.

Fraser, C., 2013. Automatic Camera Calibration in Close Range Photogrammetry. Photogrammetric Engineering and Remote Sensing 79(4), pp. 381-388.

Geiger, A., Lenz, P. and Urtasun, R., 2012. Are we ready for autonomous driving? the kitti vision benchmark suite. In: Conference on Computer Vision and Pattern Recognition (CVPR).

glassBYTEs.com, 2015. Belron's u.k. company autoglass $\AA$ launches adas calibration protocol. Website, http://www.glassbytes.com/2015/10/belrons-u-k-companyautoglass-launches-adas-calibration-protocol/. 2017-01-30.

Gruen, A. and Beyer, H. A., 2001. System Calibration Through Self-Calibration. Springer Berlin Heidelberg, Berlin, Heidelberg, pp. 163-193.

Hanel, A. and Stilla, U., 2017. Calibration of a vehicle camera system with divergent fields-of-view in an urban environment. In: 37. Wissenschaftlich-Technische Jahrestagung der DGPF.

Hartley, R. I. and Zisserman, A., 2004. Multiple View Geometry in Computer Vision. Second edn, Cambridge University Press, ISBN: 0521540518 . 
Häming, Klaus, P. G., 2010. The structure-from-motion reconstruction pipeline - a survey with focus on short image sequences Kybernetika 46(5), pp. 926-937.

Jain, A., Koppula, H. S., Raghavan, B. and Saxena, A., 2015. Know before you do: Anticipating maneuvers via learning temporal driving models. CoRR.

Kazik, T., Kneip, L., Nikolic, J., Pollefeys, M. and Siegwart, R., 2012. Real-time $6 \mathrm{~d}$ stereo visual odometry with non-overlapping fields of view. In: 2012 IEEE Conference on Computer Vision and Pattern Recognition, pp. 1529-1536.

Koenderink, J. J., Van Doorn, A. J. et al., 1991. Affine structure from motion. JOSA A 8(2), pp. 377-385.

Lowe, D. G., 1999. Object recognition from local scale-invariant features. In: Proceedings of the International Conference on Computer Vision-Volume 2 - Volume 2, ICCV '99, IEEE Computer Society, Washington, DC, USA.

Nikon, 2007. Nikon / imaging products / specifications - nikon d3. Website, http://imaging.nikon.com/lineup/dslr/d3/spec.htm. 2017-01-31.

OpenCV, 2017. Camera Calibration and 3D Reconstruction. Website, http://opencv.org. 2017-01-30.

Pemble, C. A., 2017. Garmin VIRB Ultra 30 Technical Specifications. Website, http://www8.garmin.com/automotive/pdfs/VIRBUltra30-specs.pdf. 2017-01-29.

Samuelsson, H., 2017. Vision 2020 | Volvo Cars. Website, http://www.volvocars.com/intl/about/our-stories/made-bysweden/vision-2020. 2017-01-30.

Schneider, C.-T., Bösemann, W. and Godding, R., 2017. AICON 3D Systems GmbH. Software Aicon 3d Studio.

Schneider, J. and Förstner, W., 2013. Bundle adjustment and system calibration with points at infinity for omnidirectional camera systems. Z. f. Photogrammetrie, Fernerkundung und Geoinformation 4, pp. 309-321.

Schwalbe, E., 2005. Geometric modelling and calibration of fisheye lens camera systems. In: Proceedings 2nd Panoramic Photogrammetry Workshop, Int. Archives of Photogrammetry and Remote Sensing, pp. 5-8

Sturm, P. F. and Triggs, B., 1996. A factorization based algorithm for multi-image projective structure and motion. In: Proceedings of the 4th European Conference on Computer VisionVolume II - Volume II, ECCV '96, Springer-Verlag, London, UK, UK, pp. 709-720.

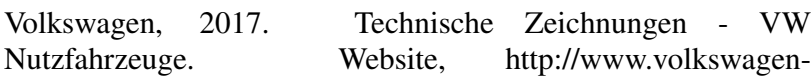
nutzfahrzeuge.ch/de/beratung - verkauf/auf-undumbauten/technische-zeichnungen.html. 2017-01-29.

Volvo, 2014. Sensor for Driver State Estimation Image, https://www.media.volvocars.com/global/en$\mathrm{gb} / \mathrm{media} /$ pressreleases/140898/volvo-cars-conducts-researchinto-driver-sensors-in-order-to-create-cars-that-get-to-knowtheir-driv. 2017-01-24.

Wu, C., 2007. A GPU implementation of Scale Invaraint Feature Transform (SIFT). Website, http://cs.unc.edu/ ccwu/siftgpu. 2017-01-10.
Wu, C., 2011. VisualSFM: A Visual Structure from Motion System. Website, http://ccwu.me/vsfm/. 2017-01-10.

Wu, C., Agarwal, S., Curless, B. and Seitz, S., 2011. Multicore bundle adjustment. In: Proc. IEEE Conf. on Computer Vision and Pattern Recognition, pp. 3057-3064 